\title{
Efficient filter design against interrupted sampling repeater jamming for wideband radar
}

\author{
Wei Xiong ${ }^{1,2}$, Gong Zhang ${ }^{1 *}$ and Wenbo Liu $^{3}$
}

\begin{abstract}
Interrupted sampling repeater jamming (ISRJ) is an effective coherent jamming, which can induce false high-resolution range profile (HRRP) and image to greatly complicate the target detection and recognition of wideband radar. To counter ISRJ, an efficient filter is designed based on the time-frequency (TF) characteristic differences between target echo and ISRJ. Meanwhile, a feasible jamming suppression scheme is proposed, which not only could eliminate ISRJ but also could reserve the target echo. The main emphasis is that the received echo suppressed by our scheme is similar with the true target's HRRP on the basis of high correlation coefficient (CC) and large signal-jamming-to-noise ratio (SJNR) improvement factor. Simulation results are covered to illustrate the feasibility and validness of jamming suppression.
\end{abstract}

Keywords: Interrupted sampling repeater jamming (ISRJ), Wideband radar, Jamming suppression, Time-frequency characteristics

\section{Introduction}

Considerable attention has been paid to the electromagnetic jamming and anti-jamming for radar system in the electronic countermeasure (ECM) community over the past two decades [1-5]. To prevent or complicate the radar target detection and recognition, much work has been done on the jamming techniques, especially some new jamming tricks that appear to counter the wideband radar system, such as the interrupted sampling repeater jamming (ISRJ) [1-9], false-target digital image synthesizer (DIS) [10-12], and scatter wave jamming (SWJ) [13-15]. As a coherent repeater jamming, ISRJ firstly proposed by Wang et al. [1] can induce a train of false targets after the pulse compression (PC) without completely sampling and storing the whole radar pulse. The method can be used by a digital radio frequency memory (DRFM)-based repeater jammer and has received growing attention from ECM community for its excellent jamming performances and advantages. Wang et al. [1] analyzed the relationship between ISRJ's parameters and jamming effects. Besides, the time-

\footnotetext{
* Correspondence: gzhang@nuaa.edu.cn

${ }^{1}$ Key Laboratory of Radar Imaging and Microwave Photonics, Ministry of Education, Nanjing University of Aeronautics and Astronautics, Nanjing 211106, People's Republic of China

Full list of author information is available at the end of the article
}

domain, frequency-domain, and amplitude characteristics of ISRJ-based false targets were described in [2]. The induced false targets make it more difficult to detect and recognize the true target. Moreover, the jamming signal could cancel the echo of true target. Feng et al. [4] explored the three conditions that should be met for the cancelation of target echo, including the repeater power, delayed time, and repeater frequency. Moreover, since the false targets induced by ISRJ always contain the phase variation information for cross-range resolution, the jamming trick also has been employed to generate two-dimensional false image and has showed its potential against radar imaging [5-9]. Wang and Cai [5] proposed the intermittent sampling jamming to counter the bi- and multistatic synthetic aperture radars (SARs). To counter the inverse SAR (ISAR), a subNyquist sampling jamming was proposed with the compressive sensing (CS)-based reconstruction algorithm [6]. Meanwhile, Pan et al. [7] investigated a CS-based matched filtering algorithm and applied it to the high-resolution range profile (HRRP) reconstruction of sub-Nyquist sampling jamming. In view of the induced false HRRP and image, ISRJ provides a complex countermeasure against the wideband radar to detect and recognize target.

Fortunately, several anti-jamming techniques have been well studied to counter the repeater jamming [15-18]. 
The very effective electronic counter-countermeasure (ECCM) for radar system is pulse diversity [16, 17], which is often adopted to combat the DRFM-based repeater jammer. However, to the best of our knowledge, most strategies are designed for the whole pulse sampling jamming including the pulse diversity, while the schemes for ISRJ might be fewer. Gong et al. [18] proposed an ECCM scheme to suppress the ISRJ for de-chirping ISAR and investigated the suppression effects for single point target. However, the strategy was only based on the ISRJ model of single point target, and the interrupted pulse samples were repeated once. The jamming suppression effects on different orders, delayed time, jamming-to-signal ratio (JSR), and signal-to-noise ratio (SNR) were not considered. Besides, suppression results were depicted but not evaluated. Moreover, the jamming suppression effect of approach $I$ relies on the time width selection of frequency smoothing window. Some jamming residue affects the suppression performances.

It is noticed that the target for wideband radar, which is also called as extended target, is represented as a series of scatterers instead of a single point. Compared with the ISRJ of single point target, the ISRJ of extended target is more complicated followed with the target scattering. It is also essential to generate the false HRRP or image against the wideband radar. In this work, we focus on the ISRJ against wideband de-chirping linear frequency modulation (LFM) radar, put forward an efficient filter for the extended target on the principles of timefrequency (TF) characteristic differences via short-time Fourier transform (STFT). Compared with the previous contributions [18], our work is proved to have the following improvements. (1) The proposed filter expands the jamming suppression approach for the extended target and is more suitable for the ISRJ against wideband radar. (2) The TF characteristic differences between target echo and ISRJ are summarized and analyzed in detail. (3) We expand the analysis of jamming suppression effects on ISRJ with different orders, delayed time, duty ratio, JSR, and SNR. (4) Correlation coefficient (CC) and signal-jamming-to-noise ratio (SJNR) improvement factor are adopted to evaluate the jamming suppression performances. (5) The overall jamming suppression scheme is depicted further. The designed filter not only could suppress the jamming and noise but also would reserve the target echo. Besides, the suppressed returns have high HRRP similarity with the target echo. It is beneficial for the target detection and recognition of wideband radar.

The remainder of our paper is structured as follows. In Section 2, the principles of ISRJ are first introduced. Then, we describe the signal model of extended target with ISRJ in Section 3. Section 4 addresses the designed filter. Meanwhile, two assessment factors of jamming suppression are depicted and the overall suppression scheme is proposed further. In Section 5, numerous simulations have been carried out and simulation results are presented, whereas conclusion is given in Section 6.

\section{Principles of ISRJ}

ISRJ is apt to be carried out, and the jamming style is feasible. The trick of ISRJ has been employed to confuse the wideband radar and protect the important military target, such as the fighter, seeker, and other air vehicles.

As shown in Fig. 1, it is assumed that the radar and escorted jammer are relatively stationary, the radar is located at $O$, the target is located at $G$ decided by the vector $R_{t}$, and the escorted jammer is located at $J$ decided by the vector $R_{j}$. The vector from the protected target to the jammer is $R_{t}$, and the included angle is denoted as $\alpha$. Herein, the point-scatter model is usually employed to model a man-made target. Suppose that the target consists of $N_{t}$ scatterers, and each scatterer is with the position $\left(x_{i}, y_{i}\right)$, complex scattering coefficient $\beta_{i}$, and vector $r_{i}$, which is relative to the reference point of target. The dashed arrow lines represent the paths of electromagnetic propagation. When the target is irradiated with the wideband radar wave, the target echo after scattering is firstly intercepted, interrupted, and stored by a preposition jammer. Then, the interrupted samples are repeated once or several times and transmitted to the radar. Besides, the amplitude modulation is sometimes necessary. Finally, the ISRJ and true target's echo is all received by the radar receiver along the radar line-ofsight (RLOS).

Let $N_{r}$ denote the repeater times, which is also called orders [2, 4]. Generally, there are three types of repeater. They are direct repeater jamming, repetitive repeater jamming, and circular repeater jamming. As depicted in Fig. 2, the interrupted sampling function of DRFM-based repeater jammer can be depicted as a rectangle envelope pulse train $\mathbf{p}(t)$ with the duration $\tau$ and interval $T_{s}[1]$

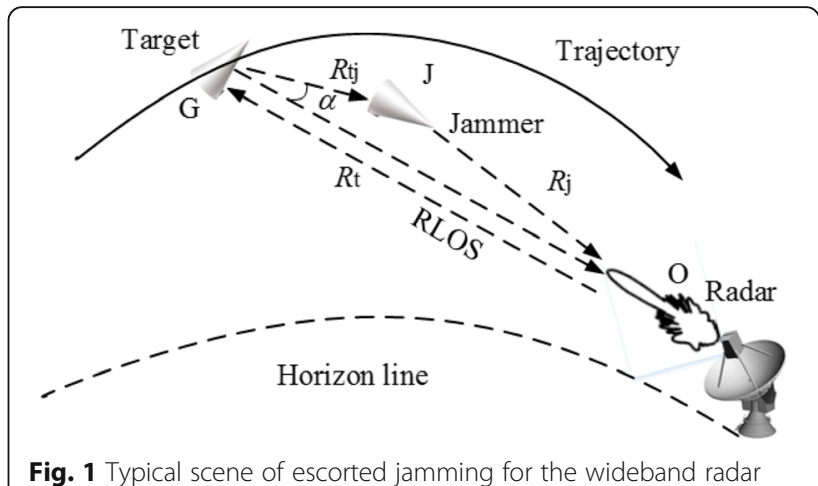




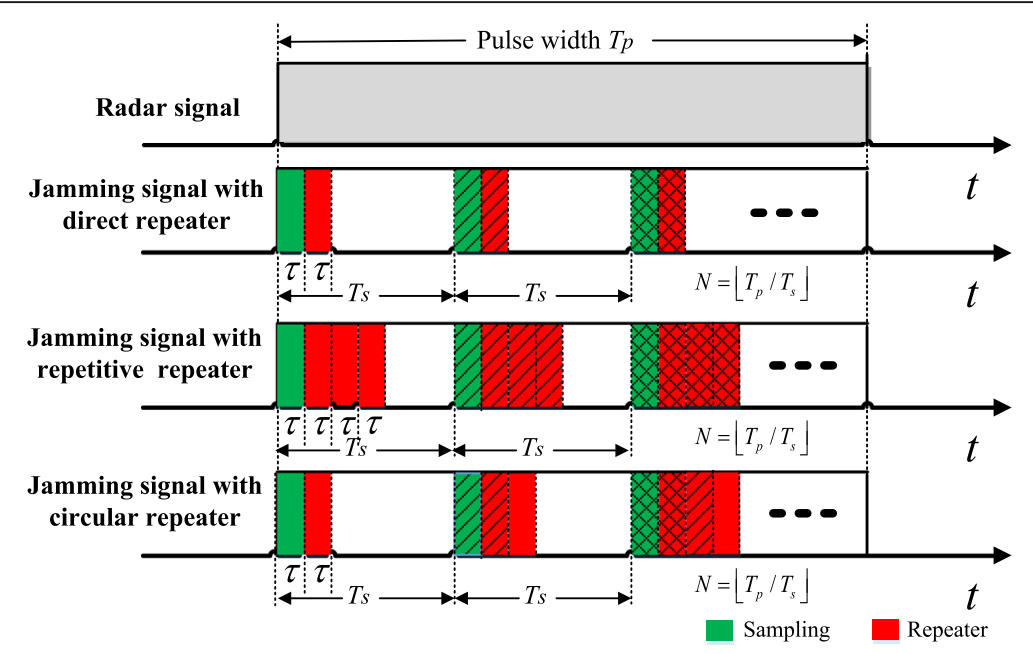

Fig. 2 An illustration of the three types of ISRJ

$$
\mathbf{p}(t)=\operatorname{rect}_{0}\left(\frac{t}{\tau}\right) * \sum_{n=-\infty}^{\infty} \delta\left(t-n T_{s}\right)
$$

where $\operatorname{rect}_{0}(t / \tau)$ is a rectangle envelope pulse, which is 1 for $0 \leq t \leq \tau$ and 0 otherwise; " stands for the convolution operation; and $\delta(\cdot)$ refers to the delta function. The duty ratio is defined as $D_{r}=\tau / T_{s}$. The direct repeater jamming immediately modulates and repeats the interrupted samples only once, whereas for the repetitive repeater jamming, the repeaters take place in the current interval $T_{s}^{i}$ several times. With regard to the circular repeater jamming, the interrupted samples in the current $T_{s}^{i}$ and the ones in the last $T_{s}^{j}(j=1,2, \cdots, i)$ are all restored, modulated, and repeated with a fixed circular order in the interval $T_{s}^{i}-\tau$. Compared with the first two types of ISRJ, the circular one has some extra requirements, such as more restored space and more random repeater order. It makes the DRFM-based repeater jammer more complicated. The direct repeater jamming and repetitive one are more common, and their suppression performances have been investigated in this paper.

\section{Signal model}

Without loss of generality, a de-chirping LFM radar is considered and the transmitted signal $\mathbf{s}\left(\hat{t}, t_{m}\right)$ is defined by

$$
\mathbf{s}\left(\hat{t}, t_{m}\right)=\operatorname{rect}\left(\frac{\hat{t}}{T_{p}}\right) \exp \left(j 2 \pi\left(f_{0} t+\frac{1}{2} K \hat{t}^{2}\right)\right),
$$

where $\operatorname{rect}\left(\hat{t} / T_{p}\right)$ represents a rectangle envelope pulse, which is 1 for $|\hat{t}| \leq T_{p} / 2$ and 0 otherwise; $T_{p}$ is the pulse width; $f_{0}$ refers to the radar carrier frequency; and $K$ is the chirp rate. Let $T_{\mathrm{PRI}}$ denote the pulse repeat interval (PRI). $t_{m}=m T_{\mathrm{PRI}}$ and $\hat{t}=t-t_{m}$ stand for the slow time and fast time, respectively [19]. When the bandwidth of radar signal $B$ is large, the target echo is always considered as the coherent summation of complex time returns from target scatterers and is expressed as

$$
\begin{aligned}
\mathbf{s}_{T}\left(\hat{t}, t_{m}\right)= & \sum_{i=1}^{N_{t}} \beta_{i} \operatorname{rect}\left(\frac{\hat{t}-2 R_{t}^{i} / c}{T_{p}}\right) \\
& \exp \left\{j 2 \pi\left[f_{0}\left(t-2 R_{t}^{i} / c\right)+\frac{1}{2} K\left(\hat{t}-2 R_{t}^{i} / c\right)^{2}\right]\right\},
\end{aligned}
$$

where $R_{t}^{i}=R_{t}+r_{i}$ is the radical distance between target's $i$ th scatterer and radar antenna. Then, the interrupted samples $\mathbf{I}_{s}\left(\hat{t}, t_{m}\right)$ of the radar transmitted signal can be regarded as [1]

$$
\mathbf{I}_{s}\left(\hat{t}, t_{m}\right)=\mathbf{p}(t) \mathbf{s}_{T}\left(\hat{t}, t_{m}\right) .
$$

Without consideration of the jammer system's inherent delay (about tens of nanosecond), the ISRJ signal for repeater can be written as

$$
\begin{aligned}
& \mathbf{s}_{J}^{0}\left(\hat{t}, t_{m}\right)=\eta_{\mathrm{JSR}} \sum_{n=0}^{N_{j^{-}} 1} \sum_{i=1}^{N_{t}} \beta_{i} \operatorname{rect}\left(\frac{\hat{t}-\tau_{\Delta J}^{i}-n T_{s}-\tau_{d}^{n}}{\tau}\right) \\
& \quad \exp \left\{j 2 \pi\left[f_{0}\left(t-\tau_{\Delta J}^{i}-n T_{s}-\tau_{d}^{n}\right)+\frac{1}{2} K\left(\hat{t}-\tau_{\Delta J}^{i}-n T_{s}-\tau_{d}^{n}\right)^{2}\right]\right\},
\end{aligned}
$$

where $\eta_{\mathrm{JSR}}$ is the JSR-based coefficient, $\tau_{\Delta J}^{i}=\left(R_{j}+R_{t j}^{i}+\right.$ $\left.R_{t}^{i}\right) / c$ is the jamming propagation delay for the $i$ th scatterer, $R_{t j}^{i}=R_{t j}+r_{i}$ denotes the radical distance between jammer's receiver and target's $i$ th scatterer, $N_{j}=\left\lfloor T_{p} / T_{s}\right\rfloor$ refers to the generated integer of a radar pulse by intermittent sampling, $L \cdot\lrcorner$ means the rounding operation toward negative infinity, and the time delay $\tau_{d}^{n}$ of the $n$th 
( $\left.n=0,1, \cdots, N_{j}-1\right)$ interrupted sampling is considered as same, i.e., $\tau_{d}^{i}=\tau_{d}^{j}=\tau_{d k}(i \neq j)$. Followed with $N_{r}$ times of repeaters, the ISRJ arrives at

$$
\mathbf{s}_{J}\left(\hat{t}, t_{m}\right)=\sum_{k=0}^{N_{r}-1} \mathbf{s}_{J}^{0}\left(\hat{t}-k \tau, t_{m}\right)
$$

It is seen that ISRJ also repeats the phase and azimuth direction information of the target echo, which makes the induced false targets would have the similar structure and attitude with the true target.

Furthermore, let $\mathbf{N}_{0}$ denote the additive noise in the range cells, then the total received echo is given as

$$
\mathbf{s}_{\text {echo }}\left(\hat{t}, t_{m}\right)=\mathbf{s}_{T}\left(\hat{t}, t_{m}\right)+\mathbf{s}_{J}\left(\hat{t}, t_{m}\right)+\mathbf{N}_{0}
$$

For the de-chirping radar, the stretch trick is always employed to process the LFM waveform with large bandwidth and obtain the PC result. It loosens the requirement for sampling frequency and can obtain highrange resolution by mixing the echo with the radar reference signal. Herein, the reference signal $\mathbf{s}_{r}\left(\hat{t}, t_{m}\right)$ can be expressed as Eq. (8):

$$
\begin{aligned}
\mathbf{s}_{r}\left(\hat{t}, t_{m}\right) & =\mathbf{s}\left(\hat{t}-t_{\text {ref }}, t_{m}\right) \\
& =\operatorname{rect}\left(\frac{\hat{t}-t_{\text {ref }}}{T_{\text {ref }}}\right) \exp \left(j 2 \pi\left(f_{0}\left(t-t_{\text {ref }}\right)+\frac{1}{2} K\left(\hat{t}-t_{\text {ref }}\right)^{2}\right)\right),
\end{aligned}
$$

where $t_{\mathrm{ref}}=2 R_{r} / c, R_{r}$ are the reference time and range, respectively. Then, the mixed target echo and ISRJ signal for the repeater arrive at the formulas as

$$
\begin{aligned}
\tilde{\mathbf{s}}_{T}\left(\hat{t}, t_{m}\right)= & \sum_{i=1}^{N_{t}} \beta_{i} \operatorname{rect}\left(\frac{\hat{t}-2 R_{t}^{i} / c}{T_{p}}\right) \\
& \exp \left[\frac{-j 4 \pi K R_{\Delta t}^{i}}{c}\left(\hat{t}-\frac{2 R_{t}^{i}}{c}\right)-\frac{j 4 \pi f_{0} R_{\Delta t}^{i}}{c}+j \pi K\left(\frac{2 R_{\Delta t}^{i}}{c}\right)^{2}\right],
\end{aligned}
$$

$$
\begin{aligned}
& \tilde{\mathbf{s}}_{J}^{0}\left(\hat{t}, t_{m}\right)=\eta_{\mathrm{ISR}} \sum_{n=0}^{N_{j}-1} \sum_{i=1}^{N_{t}} \beta_{i} \operatorname{rect}\left(\frac{\hat{t}-2 R_{j}^{i}(n) / c}{\tau}\right) \\
& \cdot \exp \left[-\frac{j 4 \pi K R_{\Delta j}^{i}(n)}{c}\left(\hat{t}-\frac{2 R_{r}}{c}\right)-\frac{j 4 \pi f_{0} R_{\Delta j}^{i}(n)}{c}+j \pi K\left(\frac{2 R_{\Delta j}^{i}(n)}{c}\right)^{2}\right],
\end{aligned}
$$

where $R_{\Delta t}^{i}=R_{t}^{i}-R_{r}, R_{\Delta j}^{i}(n)=R_{j}^{i}(n)-R_{r}$, and $R_{j}^{i}(n)=\left(R_{j}+\right.$ $\left.R_{t j}^{i}+R_{t}^{i}\right) / 2+c\left(n T_{s}+\tau_{d}^{n}\right) / 2$. Further, the mixed ISRJ with $N_{r}$ times of repeaters is given as

$$
\begin{aligned}
\tilde{\mathbf{s}}_{J}\left(\hat{t}, t_{m}\right)= & \eta_{\mathrm{ISR}} \sum_{k=0}^{N_{r}-1} \sum_{n=0}^{N_{j}-1} \sum_{i=1}^{N_{t}} \beta_{i} \operatorname{rect}\left(\frac{\hat{t}-2 R_{j}^{i}(n) / c-k \tau}{\tau}\right) \\
\cdot \exp [ & -j 2 \pi K\left(\frac{2 R_{\Delta j}^{i}(n)}{c}+k \tau\right)\left(\hat{t}-\frac{2 R_{r}}{c}\right) \\
& \left.-j 2 \pi f_{0}\left(\frac{2 R_{\Delta j}^{i}(n)}{c}+k \tau\right)+j \pi K\left(\frac{2 R_{\Delta j}^{i}(n)}{c}+k \tau\right)^{2}\right] .
\end{aligned}
$$

The stretch processing result of total echo in Eq. (7) is expressed as

$$
\tilde{\mathbf{s}}_{\text {echo }}\left(\hat{t}, t_{m}\right)=\tilde{\mathbf{s}}_{T}\left(\hat{t}, t_{m}\right)+\tilde{\mathbf{s}}_{J}\left(\hat{t}, t_{m}\right)+\tilde{\mathbf{N}}_{0} .
$$

Followed with the Fourier transform (FT), we can get the HRRP of received echo from Eq. (13), where FT(.) denotes the FT and $\tilde{\mathbf{N}}_{0}$ and $\hat{\mathbf{N}}_{0}$ refer to the stretch and FT results of additive noise $\mathbf{N}_{0}$.

$$
\begin{aligned}
\tilde{\mathbf{P}}_{\text {echo }}\left(f, t_{m}\right) & =F T\left(\tilde{\mathbf{s}}_{\text {echo }}\left(\hat{t}, t_{m}\right)\right) \\
& =\int_{\hat{t}} \tilde{\mathbf{s}}_{\text {echo }}\left(\hat{t}, t_{m}\right) \exp (-j 2 \pi f \hat{t}) d \hat{t} \\
& =\int_{\hat{t}} \tilde{\mathbf{s}}_{T}\left(\hat{t}, t_{m}\right) \exp (-j 2 \pi f \hat{t}) d \hat{t} \\
& +\int_{\hat{t}} \tilde{\mathbf{s}}_{J}\left(\hat{t}, t_{m}\right) \exp (-j 2 \pi f \hat{t}) d \hat{t}+\hat{\mathbf{N}}_{0} \\
& =\tilde{\mathbf{P}}_{T}\left(f, t_{m}\right)+\tilde{\mathbf{P}}_{J}\left(f, t_{m}\right)+\hat{\mathbf{N}}_{0} .
\end{aligned}
$$

It can be seen that the HRRP of each order false target contains the phase variation information for cross-range resolution and would further induce the false two-dimensional image. It was also analyzed in [5-9].

\section{Jamming suppression}

\subsection{TF characteristics analysis}

From Eqs. (9) and (11), it is seen that there is great similarity between the mixed true target echo and mixed ISRJ in the time domain; meanwhile, it has been analyzed that the frequency spectrum of ISRJ is a superposition of shifted replicas of the echo's frequency spectrum [1]. The traditional FT-based spectrum analysis algorithms are failed to discriminate and suppress ISRJ. However, instantaneous frequency (IF) is more suitable for characterizing the timevarying features of the signal. It provides an alternative approach to depict the characteristics of the above signal. The IF of mixed true target echo $\tilde{\mathbf{s}}_{T}$ $\left(\hat{t}, t_{m}\right)$ is given as 


$$
\begin{aligned}
\mathbf{f}_{T}(\hat{t})= & \sum_{i=1}^{N_{t}} \operatorname{rect}\left(\frac{\hat{t}-2 R_{t}^{i} / c}{T_{p}}\right) \frac{d}{d \hat{t}} \\
& {\left[-\frac{2 K R_{\Delta t}^{i}}{c}\left(\hat{t}-\frac{2 R_{t}^{i}}{c}\right)-\frac{2 f_{0} R_{\Delta t}^{i}}{c}+2 K\left(\frac{R_{\Delta t}^{i}}{c}\right)^{2}\right] } \\
= & \sum_{i=1}^{N_{t}} \operatorname{rect}\left(\frac{\hat{t}-2 R_{t}^{i} / c}{T_{p}}\right)\left(-K \frac{2 R_{\Delta t}^{i}}{c}\right) .
\end{aligned}
$$

And the IF of mixed ISRJ $\tilde{\mathbf{s}}_{J}\left(\hat{t}, t_{m}\right)$ is written as

$$
\begin{aligned}
\mathbf{f}_{J}(\hat{t}) & =\sum_{k=0}^{N_{r}-1} \sum_{n=0}^{N_{j}-1} \sum_{i=1}^{N_{t}} \operatorname{rect}\left(\frac{\hat{t}-2 R_{j}^{i}(n) / c-k \tau}{\tau}\right) \\
& \cdot \frac{d}{d \hat{t}}\left[-K\left(\frac{2 R_{\Delta j}^{i}(n)}{c}+k \tau\right)\left(\hat{t}-\frac{2 R_{r}}{c}\right)-f_{0}\left(\frac{2 R_{\Delta j}^{i}(n)}{c}+k \tau\right)\right. \\
& \left.+\frac{K}{2}\left(\frac{2 R_{\Delta j}^{i}(n)}{c}+k \tau\right)^{2}\right] \\
& =\sum_{k=0}^{N_{r}-1} \sum_{n=0}^{N_{j}-1} \sum_{i=1}^{N_{t}} \operatorname{rect}\left(\frac{\hat{t}-2 R_{j}^{i}(n) / c-k \tau}{\tau}\right)\left(-K\left(\frac{2 R_{\Delta j}^{i}(n)}{c}+k \tau\right)\right) .
\end{aligned}
$$

It can be analyzed that some characteristic differences as follows make it feasible to design a filter to suppress ISRJ, especially on the time points when the jammer receives and samples the radar signal.

(1) For the $k$ th repeater and the $i$ th scatterer, the IF of ISRJ in the pulse duration is not continuous due to $2\left[R_{j}^{i}(n+1)-R_{j}^{i}(n)\right] / c=T_{s}>\tau$, while the one of true target echo is continuous [18].

(2)For the $k$ th repeater and the $i$ th scatterer, the relative delayed time $2 R_{j}^{i}(n) / c+k \tau=\left(R_{j}+R_{t j}^{i}+R_{t}^{i}\right) / c+\left(n T_{s}+\right.$ $\left.\tau_{d}^{n}\right)+k \tau$ of ISRJ in Eq. (15) is different from the delayed time $2 R_{t}^{i} / c$ of true target echo in Eq. (14). It contributes to make the TF energy distribution of each order ISRJ shift from the one of true target echo.

(3) From Fig. 2, we can draw out that the time for interrupted sampling and repeated transmitting is divisional in the pulse duration, i.e., the receivetransmit antenna of jammer works asynchronously.

\subsection{Filter design}

On the premise of ensuring the target echo to be reserved and jamming to be suppressed, the ideal weights of filter for the range bins of target should be 1, while the ones for the other range bins should be 0 . In the subsection, an effective filter is designed to suppress ISRJ based on the analyzed TF characteristic differences.

From Eqs. (14) and (15), it is obvious that the calculated IF of mixed target echo and jamming returns are all stationary and linear. It means that the conventional liner time-frequency analysis (TFA) methods are qualified to analyze their TF characteristics, such as STFT [20] and wavelet transform (WT) [21]. In this paper, STFT is employed to extract the TF characteristics, which is established on the assumption that the considered signal is piece-wisely stationary in a short time. By performing STFT on the mixed target echo and mixed jamming returns, the energy distribution of $\mathrm{TF}$ representation can be described as

$$
\begin{aligned}
\mathbf{T F}_{T}(\hat{t}, f) & =\left|\operatorname{STFT}\left(\tilde{\mathbf{s}}_{T}\left(\hat{t}, t_{m}\right)\right)\right|^{2} \\
& =\left|\int \tilde{\mathbf{s}}_{T}\left(\hat{t}, t_{m}\right) \omega(\hat{t}-t) \exp (-j 2 \pi f \hat{t}) d \hat{t}\right|^{2}, \\
\mathbf{T F}_{J}(\hat{t}, f) & =\left|\operatorname{STFT}\left(\tilde{\mathbf{s}_{J}}\left(\hat{t}, t_{m}\right)\right)\right|^{2} \\
& =\left|\int \tilde{\mathbf{s}}_{J}\left(\hat{t}, t_{m}\right) \omega(\hat{t}-t) \exp (-j 2 \pi f \hat{t}) d \hat{t}\right|^{2},
\end{aligned}
$$

where $\operatorname{STFT}(\cdot)$ represents the STFT and $\omega(\hat{t})$ means the frequency smoothing window. Moreover, the TF energy distribution of total received echo arrives at

$$
\begin{aligned}
\mathbf{T F}_{T J}(\hat{t}, f) & =\left|\operatorname{STFT}\left(\tilde{\mathbf{s}}_{\text {echo }}\left(\hat{t}, t_{m}\right)\right)\right|^{2} \\
& =\mathbf{T F}_{T}(\hat{t}, f)+\mathbf{T F}_{J}(\hat{t}, f)+\mathbf{N}_{1},
\end{aligned}
$$

where $\quad \mathbf{N}_{1}=\left|\operatorname{STFT}\left(\tilde{\mathbf{N}}_{0}\right)\right|^{2} \quad$ represents noise energy followed with STFT.

In the time domain, we employ the same trick in [18] to find the time points with great TF characteristic differences when the jammer samples the radar signal and first sum $\mathbf{T F}_{\mathrm{TJ}}(\hat{t}, f)$ along the frequency direction and define the result as

$$
\boldsymbol{\Phi}(\hat{t})=\int_{f} \mathbf{T F}_{\mathrm{TJ}}(\hat{t}, f) d f .
$$

It is assumed that the energy concentrated domain of Eq. (19) is $\left[t_{1}, t_{2}\right]$ in the pulse duration, the time points of which satisfy that $\boldsymbol{\Phi}\left(t_{1}\right)>\zeta, \boldsymbol{\Phi}\left(t_{2}\right)>\zeta$ and $\boldsymbol{\Phi}(\hat{t})<\zeta, \hat{t} \notin$ $\left[t_{1}, t_{2}\right]$, and $\zeta$ is the energy threshold. Then, we divide the domain $\left[t_{1}, t_{2}\right]$ into $L$ segments and select the time point $\hat{t}_{l}^{\mathrm{min}}$ with the minimum value of $\boldsymbol{\Phi}(\hat{t})$ in each segment $\left[t_{l 1}, t_{l 2}\right]$ as follows:

$$
\begin{aligned}
\hat{t}_{l}^{\min }=\arg _{t} \min \Phi(\hat{t}) \quad l=1,2, \cdots, L \\
\text { s.t. }\left\{\begin{array}{l}
\hat{t} \in\left[t_{l 1}, t_{l 2}\right] \\
t_{l 1}=t_{1}+(l-1) T_{w} \\
t_{l 2}=t_{1}+l T_{w},
\end{array}\right.
\end{aligned}
$$

where $T_{w}=\left(t_{2}-t_{1}\right) / L$ is the width of each segment. The selected time points $\left\{\hat{t}_{l}^{\min }\right\}_{l=1}^{L}$ with great TF characteristic differences are considered for the interrupted sampling of jammer, i.e., the ISRJ might be absent at these 


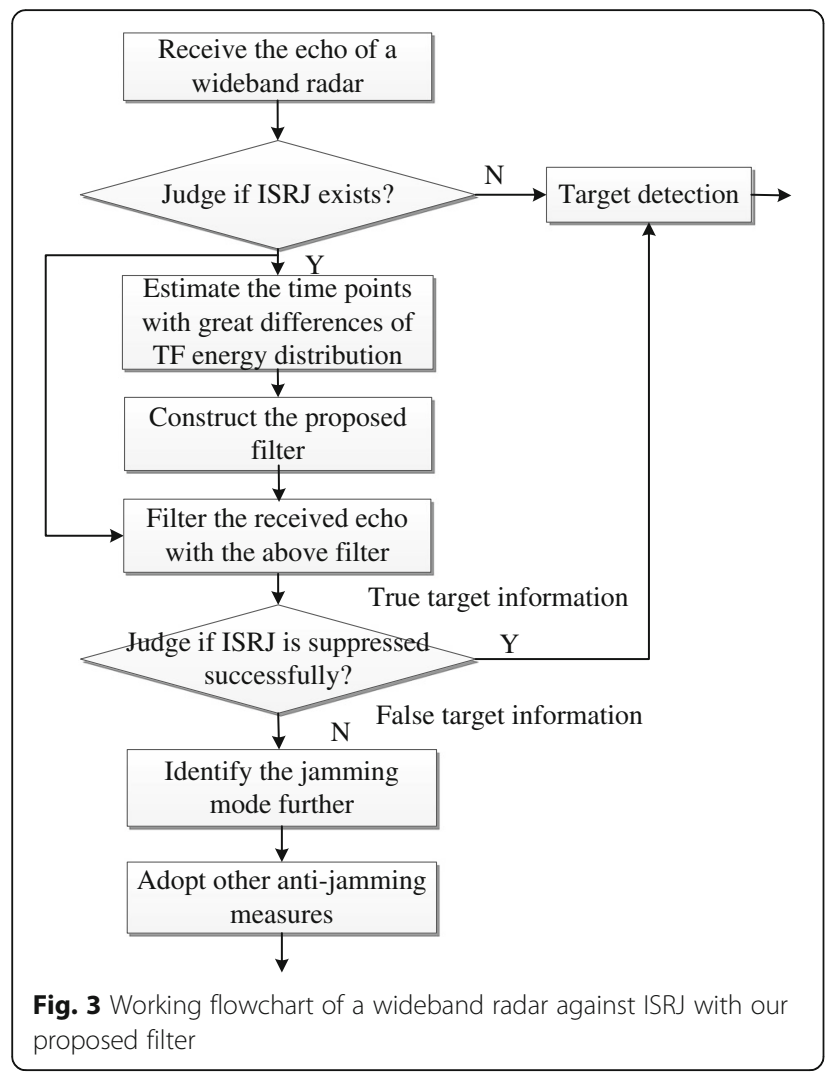

points in the mixed returns. In addition, the received echo after stretch processing near these time points could be regarded as the mixed target echo. It is extracted and represented as

$$
\mathbf{s}_{e}(\hat{t})=\sum_{l=1}^{L} \operatorname{rect}\left(\frac{\hat{t}-\hat{t}_{l}^{\min }}{T_{\text {win }}}\right) \tilde{\mathbf{s}}_{\text {echo }}\left(\hat{t}, t_{m}\right)
$$

Subsequently, we have the normalized filter $\mathbf{H}(f)$ and express it as

$$
\mathbf{H}(f)=\left|\mathrm{FT}\left(\mathbf{s}_{e}(\hat{t})\right)\right|^{2} / \kappa=\left|\int_{\hat{t}} \mathbf{s}_{e}(\hat{t}) \exp (-j 2 \pi f \hat{t}) d \hat{t}\right|^{2} / \kappa
$$

where $\kappa$ is the maximum of $\left|\mathrm{FT}\left(\mathbf{s}_{e}(\hat{t})\right)\right|^{2}$. It can be seen that the designed weights of $\mathbf{H}(f)$ for the target range bins are larger and even be 1, while the ones for the other range bins are much smaller and even be 0 . Finally, the jamming and noise are suppressed after multiplying the HRRP of the received returns by the designed filter function. It can be formulated as

$$
\mathbf{P}\left(f, t_{m}\right)=\mathbf{H}(f) \cdot \tilde{\mathbf{P}}_{\text {echo }}\left(f, t_{m}\right)=\mathbf{H}(f) \cdot\left(\tilde{\mathbf{P}}_{J}\left(f, t_{m}\right)+\tilde{\mathbf{P}}_{T}\left(f, t_{m}\right)+\hat{\mathbf{N}}_{0}\right) .
$$

\subsection{Suppression performance assessment}

Herein, the CC and SJNR improvement factor are adopted to evaluate the jamming suppression performances of our proposed algorithm.

In general, the CC between the ideal HRRP of target $\tilde{\mathbf{P}}_{T}\left(f, t_{m}\right)$ and the filtered result $\mathbf{P}\left(f, t_{m}\right)$ can be taken to evaluate the jamming suppression performances for wideband radar. It is always hoped that the expected CC would satisfy

$$
E\left(\rho\left(\mathbf{P}\left(f, t_{m}\right), \tilde{\mathbf{P}}_{T}\left(f, t_{m}\right)\right)\right) \geq \rho_{0},
$$

where

$$
E\left(\rho\left(\mathbf{P}\left(f, t_{m}\right), \tilde{\mathbf{P}}_{T}\left(f, t_{m}\right)\right)\right)=\frac{E\left(\sum_{n=1}^{N} \mathbf{P}\left(f_{n}, t_{m}\right) \tilde{\mathbf{P}}_{T}\left(f_{n}, t_{m}\right)\right)}{E\left(\left\|\mathbf{P}\left(f, t_{m}\right)\right\|_{2}\left\|\tilde{\mathbf{P}}_{T}\left(f, t_{m}\right)\right\|_{2}\right)} .
$$

$\rho_{0} \in[0,1]$ refers to the lower bound of CC (LBCC).

The other performance assessment is indicated by the SJNR improvement factor. For the wideband radar, the power of target and jamming extends some range bins. We have the improvement factor of SJNR represented as

$$
\begin{aligned}
& \delta_{\text {sinr }}=20 \log 10\left(\frac{\mathrm{Amp}_{p}^{S_{\max }}}{\mathrm{Amp}_{p}^{J_{\max }}+\mathrm{Amp}_{p}^{N_{\text {ave }}}}\right) \\
& -20 \log 10\left(\frac{\mathrm{Amp}_{\bar{p}_{\text {echo }}}^{S_{\max }}}{\mathrm{Amp}_{\bar{p}_{\text {echo }}}^{J_{\max }}+\mathrm{Amp}_{\tilde{p}_{\text {echo }}}^{N_{\text {ave }}}}\right),
\end{aligned}
$$

where $\left(\mathrm{Amp}_{p}^{S_{\max }}, \mathrm{Amp}_{p}^{J_{\max }}\right)$ and $\left(\mathrm{Amp}_{\tilde{p}_{\text {echo }}}^{S_{\max }}, \mathrm{Amp}_{\tilde{p}_{\text {echo }}}^{J_{\max }}\right)$ denote the maximum amplitudes of target and jamming in $\mathbf{P}\left(f, t_{m}\right)$ and $\tilde{\mathbf{P}}_{\text {echo }}\left(f, t_{m}\right)$, respectively. $\mathrm{Amp}_{p}^{N_{\text {ave }}}$ and

Table 1 Parameter description of the ISAR, target, and jammer

\begin{tabular}{lllll}
\hline Radar parameters & Carrier frequency $f_{0}$ & $10 \mathrm{GHz}$ & Pulse width $T_{p}$ & $128 \mu \mathrm{s}$ \\
& Band width $B$ & $1 \mathrm{GHz}$ & PRI & $1 \mathrm{~ms}$ \\
& Width of $\omega(\hat{t})$ & $4.2 \mu \mathrm{s}$ & Location of $O$ & $(0 \mathrm{~m}, 0 \mathrm{~m})$ \\
Target parameters & SNR & $25 \mathrm{~dB}$ & Location of $\mathrm{G}$ & $(0 \mathrm{~m}, 100,005 \mathrm{~m})$ \\
Jammer parameters & Repeat sampling interval $T_{s}$ & $20 \mu \mathrm{s}$ & $5 / 10 \mu \mathrm{s}$ \\
& Delayed time $\tau_{d}^{n}$ & $5.45 / 5.11 \mu \mathrm{s}$ & Sampling duration $\tau$ & 1 and 3 \\
& JSR & $20 \mathrm{~dB}$ & Order $N_{r}$ & $(0 \mathrm{~m}, 10,000 \mathrm{~m})$ \\
\hline
\end{tabular}


$\mathrm{Amp}_{\tilde{p}}^{N_{\text {ave }}} \mathrm{N}_{\text {ech }}$ refer to the average noise amplitudes before and after the jamming suppression.

\subsection{Jamming suppression working flow}

The working flowchart of wideband radar for ISRJ suppression is given in Fig. 3. When the protected target echo and ISRJ are received simultaneously, the wideband radar system will primarily judge whether ISRJ exists. It could be identified based on the representation characteristics of HRRP and SAR/ISAR image. If the ISRJ is detected and confirmed, the radar system has to extract the time points with great differences of TF energy distribution. Then, the proposed filter is constructed and the $\mathrm{PC}$ result of received returns will be fed to the filter. Finally, the jamming suppression performances will be evaluated. If the suppression result has high CC and large SJNR improvement factor, the target could be detected and recognized. If some ISRJ residue still retains with low CC and small SJNR improvement factor, the

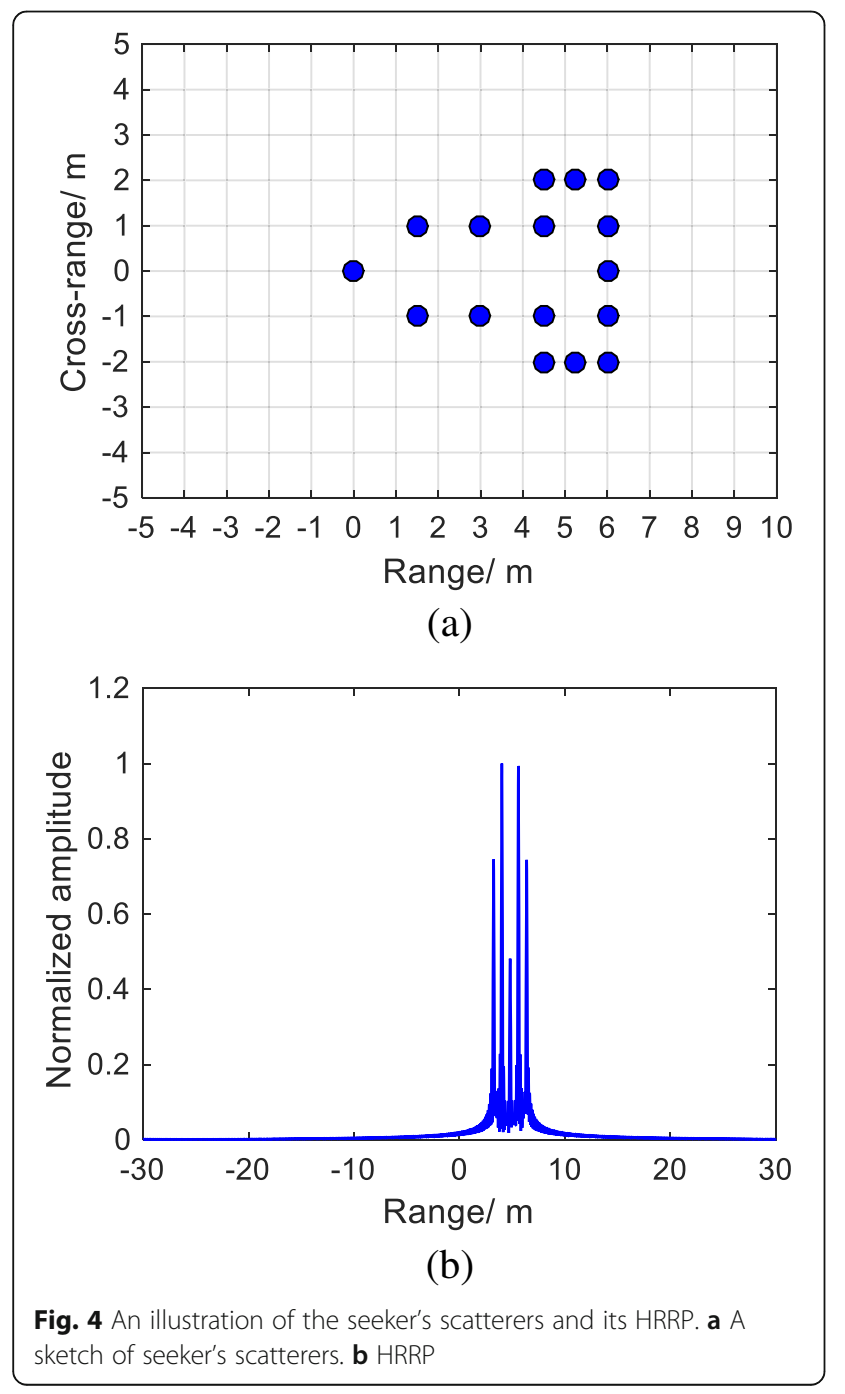

jamming mode will be identified with other characteristics and another corresponding anti-jamming measures will be adopted further, such as the trick of complicated radar waveform and some measures of low probability of interception.

\section{Simulation results and analysis}

In this work, the simulated radar system is a wideband LFM ISAR, whose parameters and target and jammer are all listed in Table 1 . These parameters are also cited in $[2,18]$. It is pointed out that the hamming window is employed as the frequency smoothing window $\omega(\hat{t})$ in Eqs. (16), (17), and (18), and the energy threshold $\zeta$ in Eq. (20) is set as 0.1 after the normalization of $\boldsymbol{\Phi}(\hat{t})$. The simulated seeker, as shown in Fig. 4a, is regarded as the extended target, which is consisted of 16 scatterers with each reflection coefficient $\beta_{i}=0.4$. The location of $G$ in Table 1 and Fig. 1 means the position of the seeker head scatterer, which is also denoted as the reference

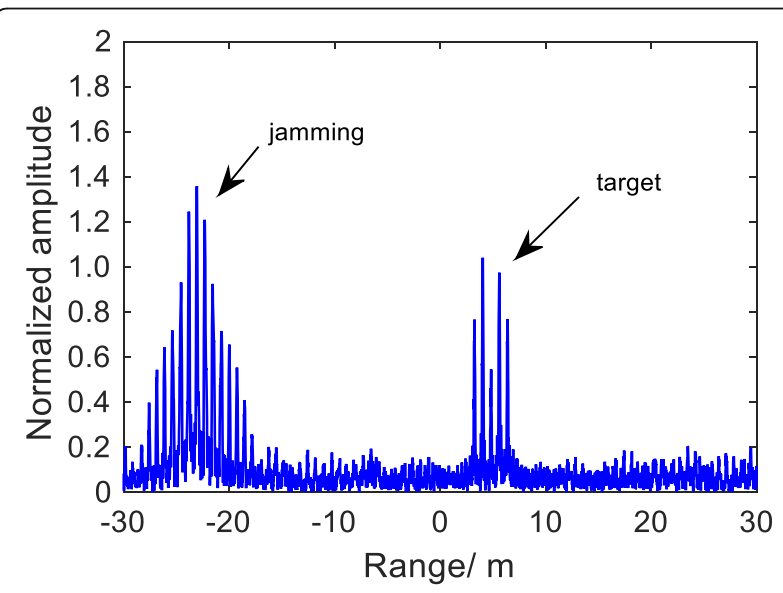

(a)

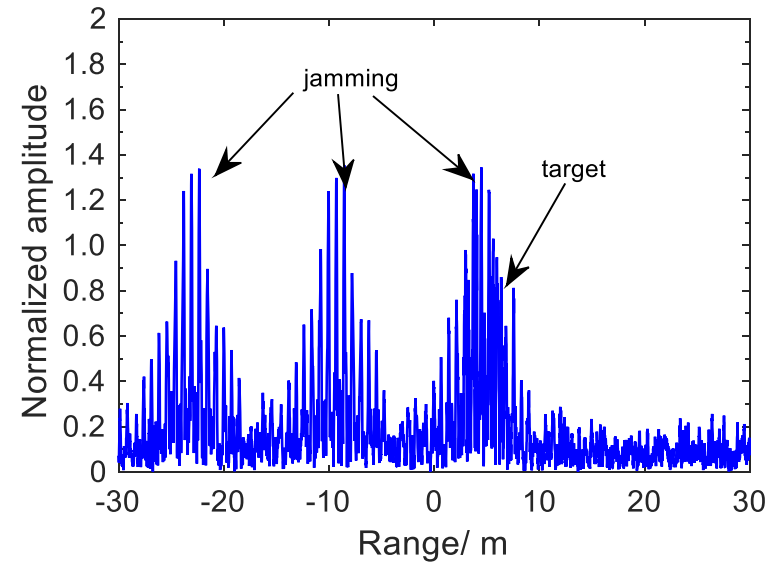

(b)

Fig. 5 HRRP of the received echo, where $\tau_{d}^{n}=5.45 \mu \mathrm{s}$. a Once repeater. $\mathbf{b}$ Thrice repeater 


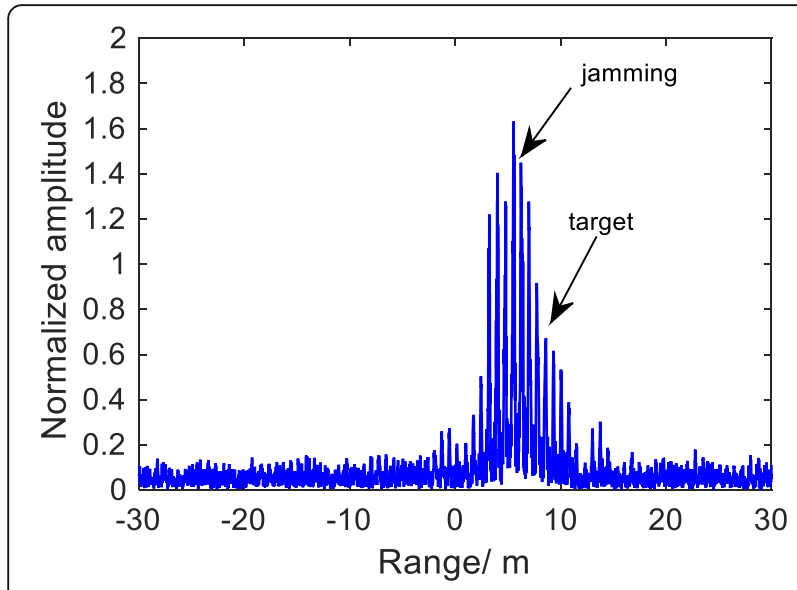

(a)

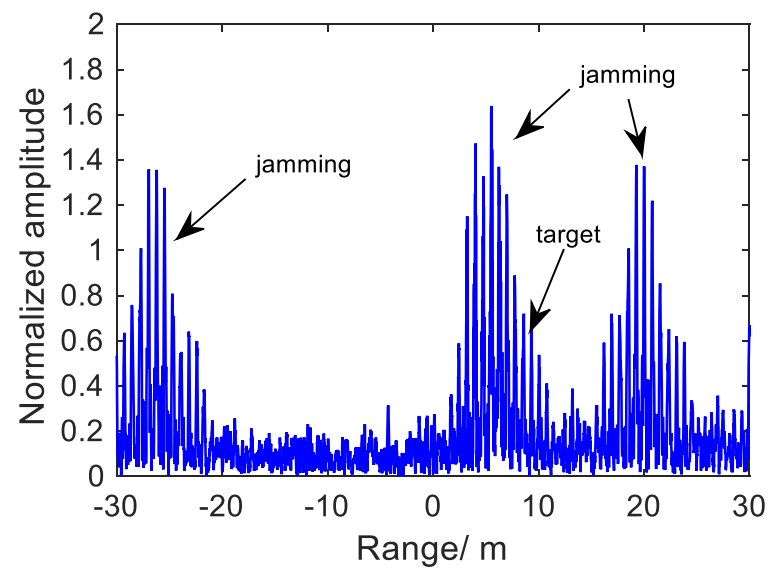

(b)

Fig. $6 \mathrm{HRRP}$ of the received echo, where $\tau_{d}^{n}=5.11 \mu \mathrm{s}$. a Once repeater. $\mathbf{b}$ Thrice repeater

origin of coordinate in Fig. 4a. At the beginning, Fig. 4b illustrates the ideal target's HRRP without jamming and noise. Then, some simulations of jamming suppression have been carried out, and suppression results are analyzed in detail.

\subsection{ISRJ effects}

In the subsection, ISRJ is first investigated against the seeker detection and recognition. When $\mathrm{SNR}=25 \mathrm{~dB}$ and $J S R=20 \mathrm{~dB}$, Fig. 5 shows the jamming effects for radar system with the delayed time $\tau_{d}^{n}=5.45 \mu$ s and different order $\left(N_{r}=1,3\right)$. Besides, the ones with delayed time $\tau_{d}^{n}=5.11 \mu$ s are depicted in Fig. 6 .

The generated HRRP of false targets also demonstrate that the number of false targets is related to the order [1-4]. In addition, it is seen that ISRJ can induce false targets or barrage jamming, especially the preposition false target, which would greatly complicate the target detection and recognition of radar system.

\subsection{Jamming suppression performances}

Herein, the jamming suppression performances of the proposed filter are presented. In view of the unknown sampling duration time of DRFM-based jammer, we just

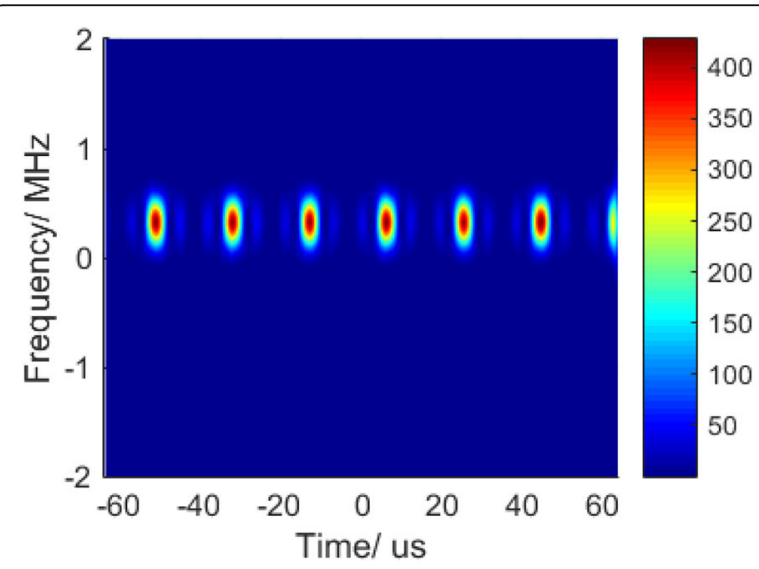

(a)

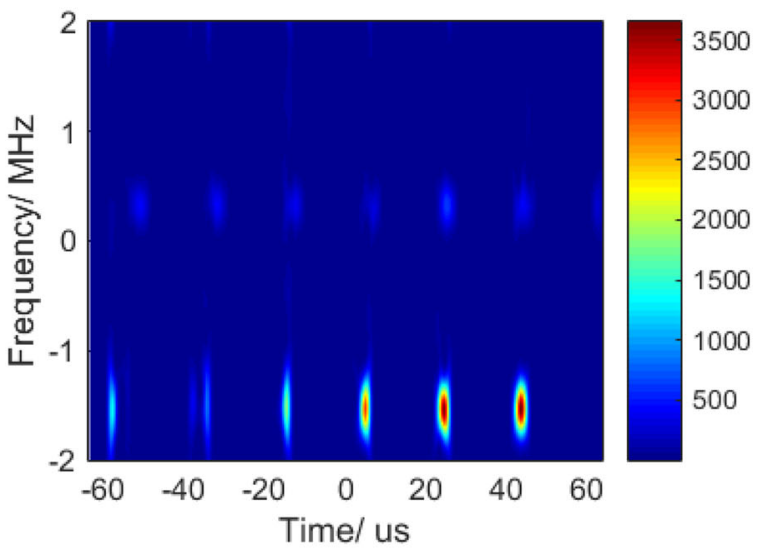

(b)

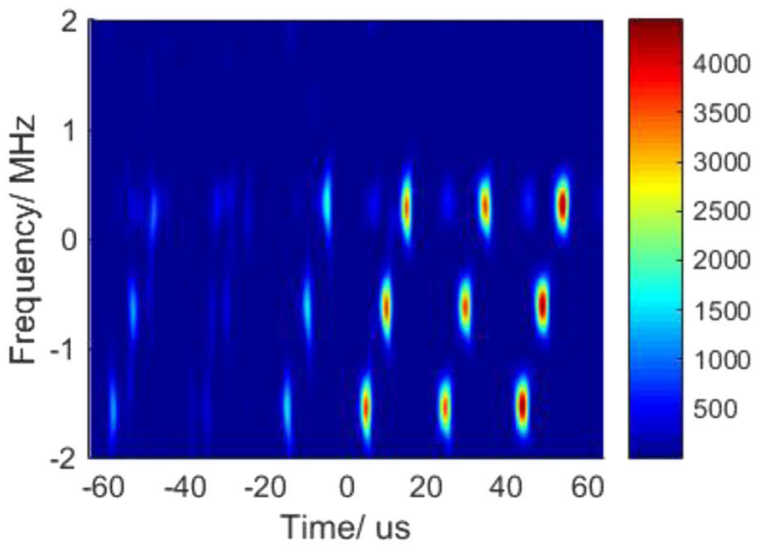

(c)

Fig. 7 Energy distribution of TF, where $\tau_{d}^{n}=5.45 \mu \mathrm{s}$. a Target echo. $\mathbf{b}$ Received returns in Fig. 5a. c Received returns in Fig. 5b 


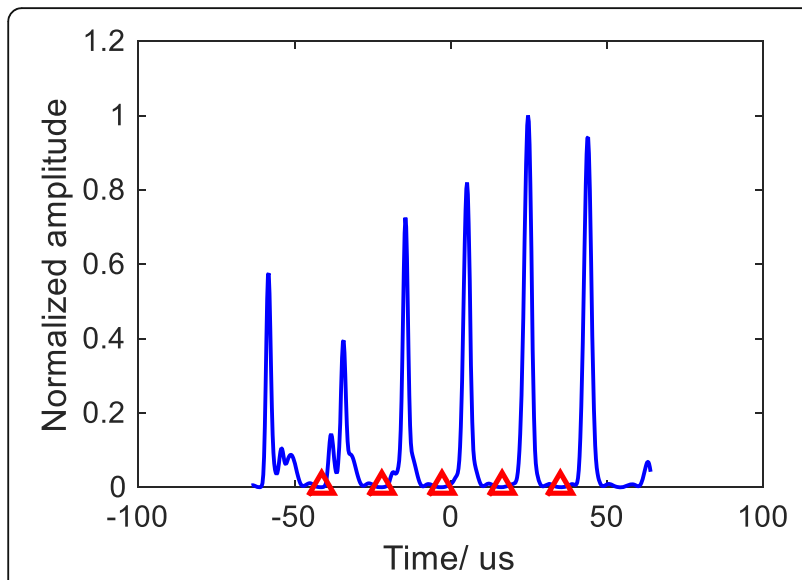

(a)

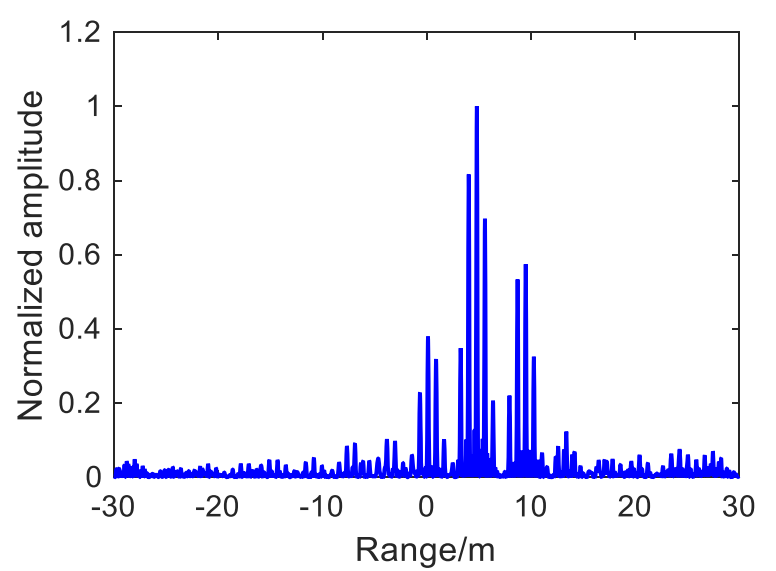

(b)

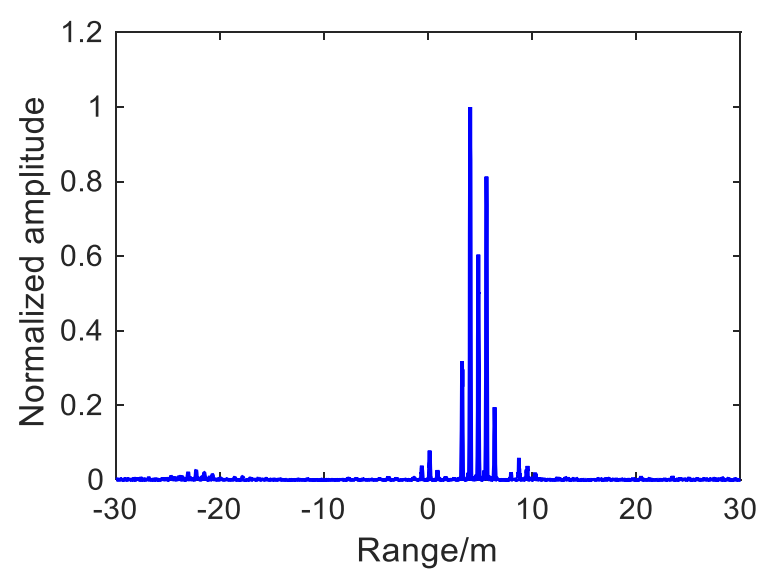

(c)

Fig. 8 Simulation results of received returns in Fig. 5a. a Selected time points. b Filter function. c Suppression result

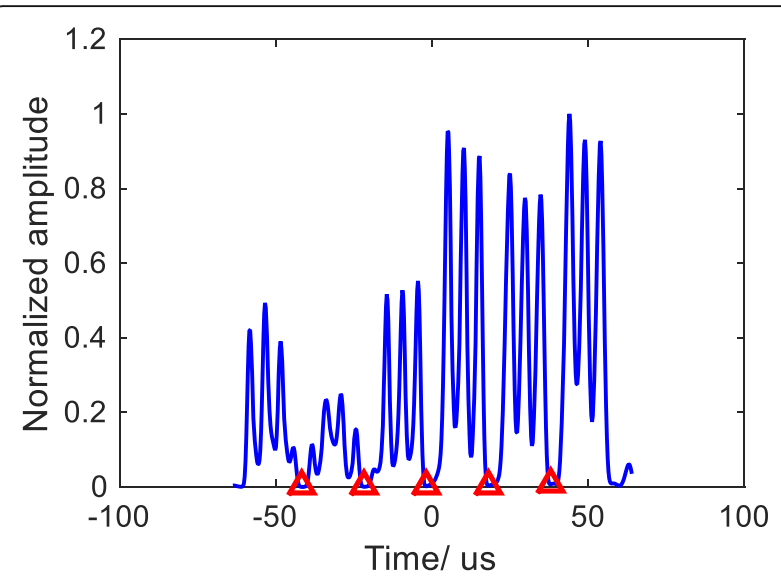

(a)

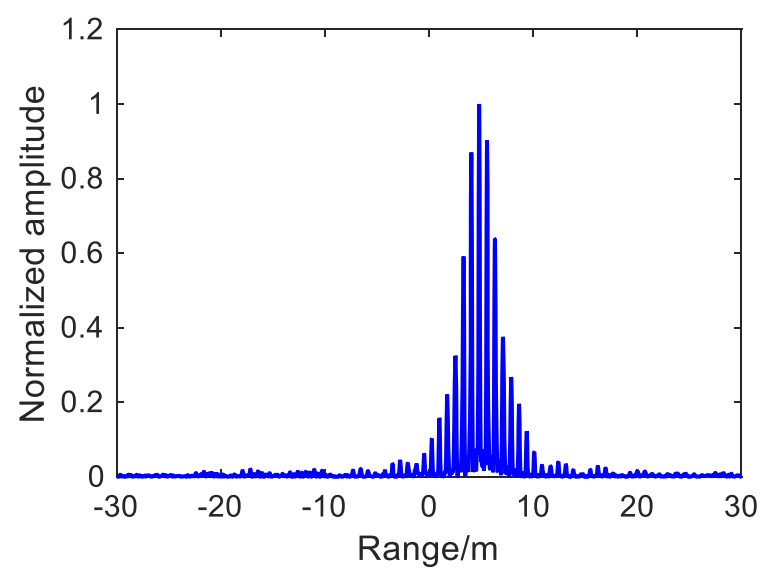

(b)

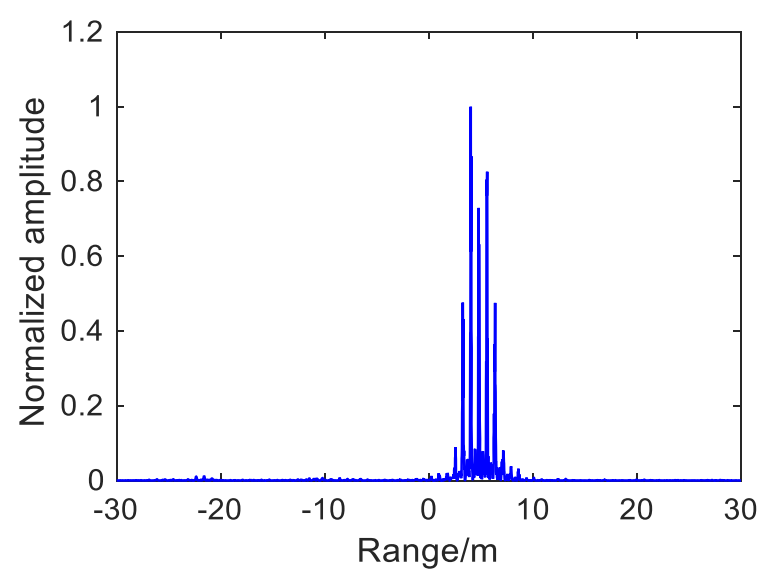

(c)

Fig. 9 Simulation results of received returns in Fig. 5b. a Selected time points. b Filter function. c Suppression result 
select single time point $\hat{t}_{l}^{\min }$ in each segment $\left[t_{l 1}, t_{l 2}\right]$ in Eq. (21). Four different cases are discussed in detail.

\subsubsection{Performances versus order}

As a representation, Fig. 7a shows the energy distribution $\mathbf{T F}_{T}(\hat{t}, f)$ of the protected target echo via STFT, while the energy distribution $\mathbf{T F}_{\mathrm{TJ}}(\hat{t}, f)$ for Fig. 5 is depicted in Fig. 7b, c. When the interrupted samples are repeated once $\left(N_{r}=1\right)$, the selected time points in Eq. (20) are symbolized by the notation " $\Delta$ " in Fig. $8 \mathrm{a}$, where the segment number is $M=5$. The filter function is shown in Fig. $8 \mathrm{~b}$, and the jamming suppression result of our proposed filter is further illustrated in Fig. 8c. Similarly, when the interrupted samples are repeated thrice $\left(N_{r}=3\right)$, the simulation results are presented in Fig. 9. We can find that not only the jamming and noise are suppressed but also the target echo is reserved effectively.

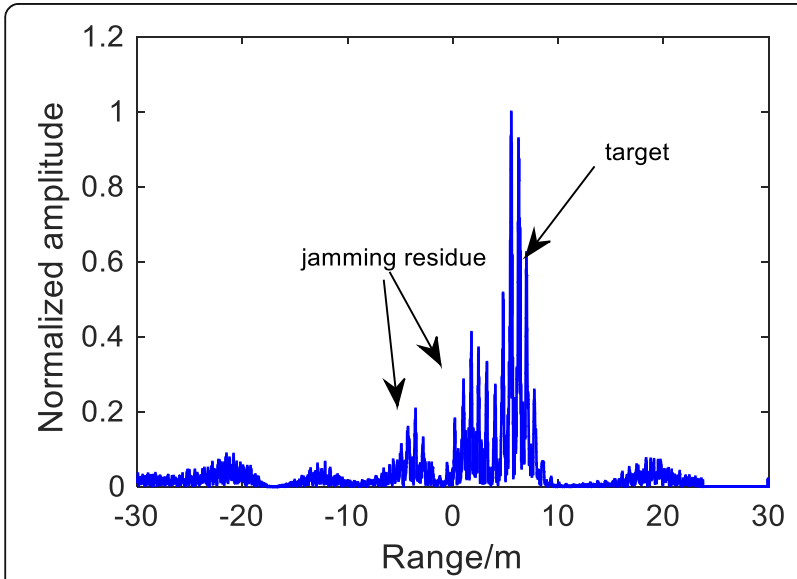

(a)

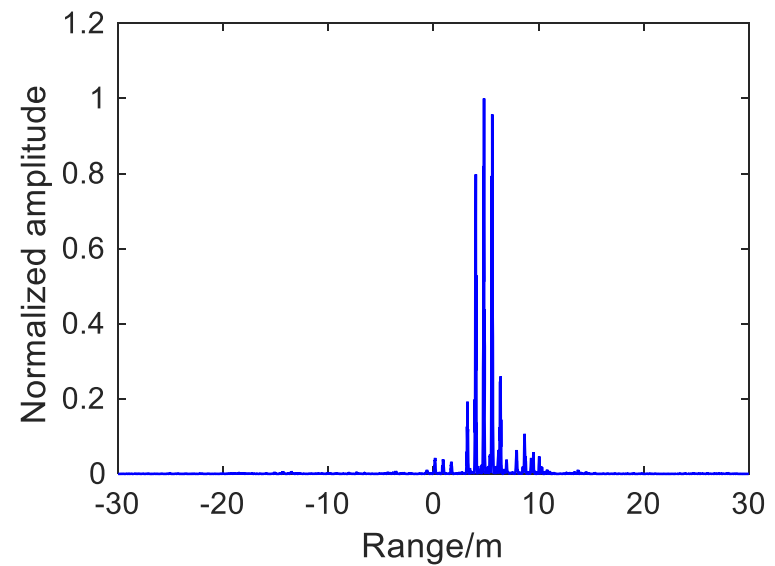

(b)

Fig. 10 Jamming suppression result comparison of the received returns in Fig. 6a. a Approach / in [18]. b Our proposed filter

\subsubsection{Performances versus delayed time}

As shown in Figs. 5 and 6, it is concluded that different delayed times would induce different effects of false target, which might be preposition, postposition, or barrage jamming. Different from case 1, the jamming suppression performances for Fig. 6a with the delayed time $\tau_{d}^{n}=$ $5.11 \mu \mathrm{s}$ are investigated. As a comparison, the jamming suppression result of approach $I$ in [18] is shown in Fig. 10a, while that of our proposed filter is shown in Fig. 10b. Since the time width of $\omega(\hat{t})$ is smaller than the sampling duration $\tau$, the spectrum of the target signal spreads by the windowing. Then, the ISRJ near the target, especially the barrage jamming, is hardly to be suppressed by the approach $I$ and some jamming residues are still reserved in Fig. 10a. From Fig. 10b, it implies that our proposed jamming suppression scheme also can obtain desired suppression performances on the

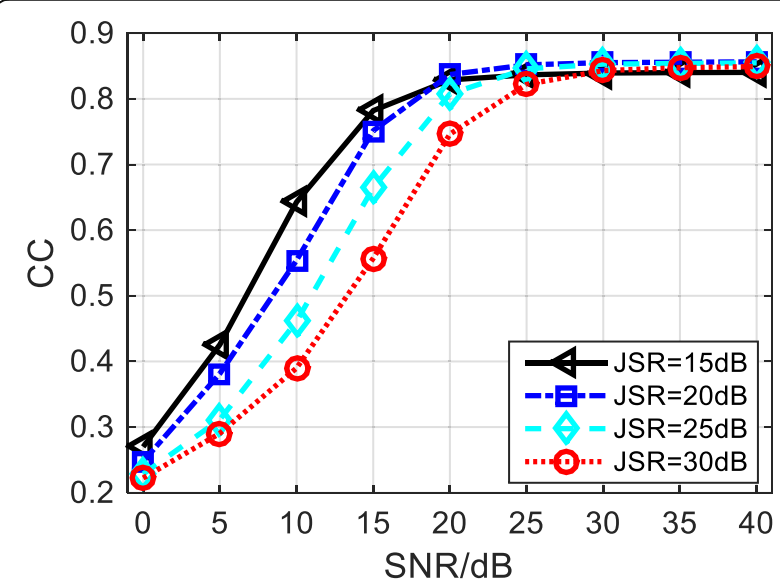

(a)

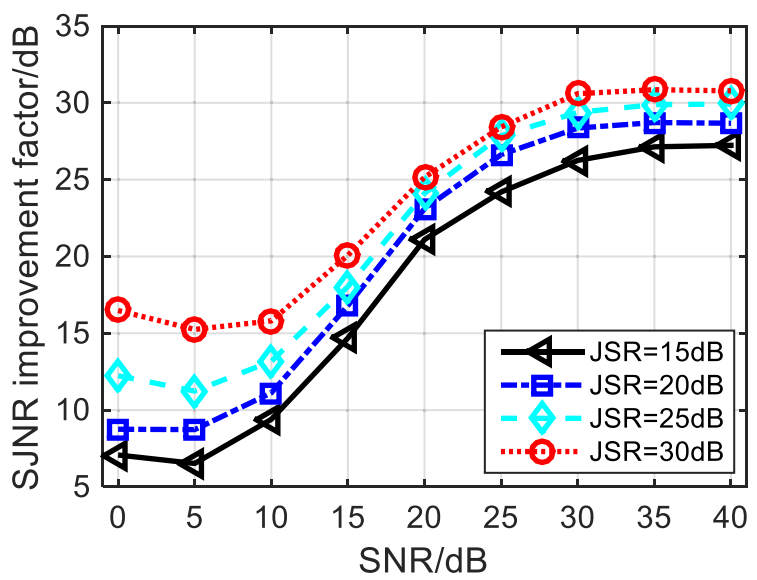

(b)

Fig. 11 Performances of jamming suppression with different JSRs and SNRs, where $\tau_{d}^{n}=5.45 \mu$ s and $N_{r}=1$. a Curves of CC. $\mathbf{b}$ Curves of SJNR improvement factor 


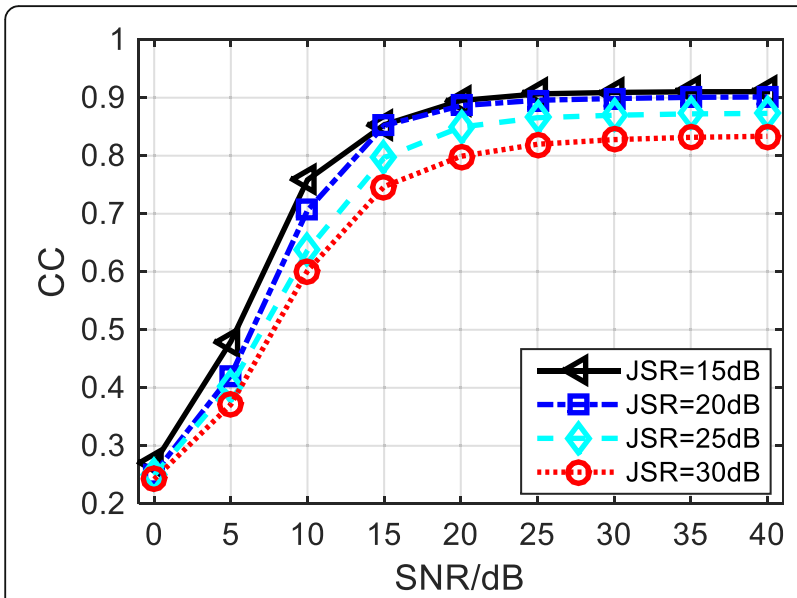

(a)

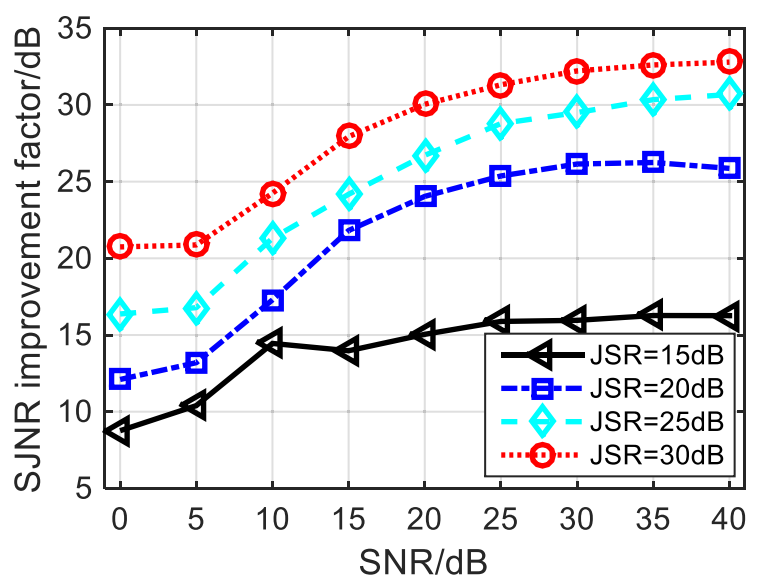

(b)

Fig. 12 Performances of jamming suppression with different JSRs and SNRs, where $\tau_{d}^{n}=5.45 \mu$ s and $N_{r}=3$. a Curves of CC. $\mathbf{b}$ Curves of SJNR improvement factor

barrage jamming in Fig. 6a, although a little of the jamming residue exists around the target range bins.

\subsubsection{Performances versus SNR and JSR}

In this case, the effects of different SNR and JSR on jamming suppression are shown. The number of Monte Carlo trials is 200 for each of the different SNR or JSR. For the case of Fig. 8, the curves of $\mathrm{CC}$ are shown in Fig. 11a, and the curves of SJNR improvement factor are presented in Fig. 11b. Analogously, Fig. 12 depicts the jamming suppression performances with different SNRs and JSRs for the case of Fig. 9. From Figs. 11 and 12, we can summarize the observations as follows:

1. The curves of CC climb observably when SNR < $25 \mathrm{~dB}$ and increase slightly when $\mathrm{SNR}>25 \mathrm{~dB}$. In Fig. 11a, the CC for each JSR is larger than 0.8 when $\mathrm{SNR}>25 \mathrm{~dB}$. In Fig. 12a, when SNR > $25 \mathrm{~dB}$, the CCs for JSR $=15$ and $20 \mathrm{~dB}$ arrive at 0.9 , while the ones for $\mathrm{JSR}=25$ and $30 \mathrm{~dB}$ are larger than 0.8. Without generality, the LBCC $\rho_{0}$ is set as 0.8. It is indicated that when SNR > $25 \mathrm{~dB}$, the received echo suppressed by our proposed scheme is similar with the HRRP of ideal target echo, which is helpful for the target detection and recognition of wideband radar.

2. The SJNR improvement factor grows in collaboration with SNR increasing and larger SJNR improvement factor can be obtained in the case of larger JSR. It means that our proposed scheme can distinctly improve the SJNR and is also beneficial to increase the effectual range of radar target detection in the complicated electromagnetic environment.

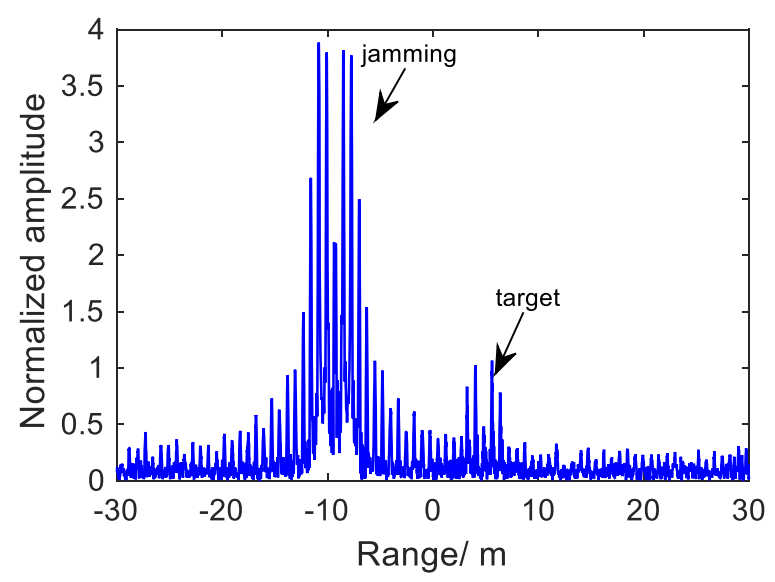

(a)

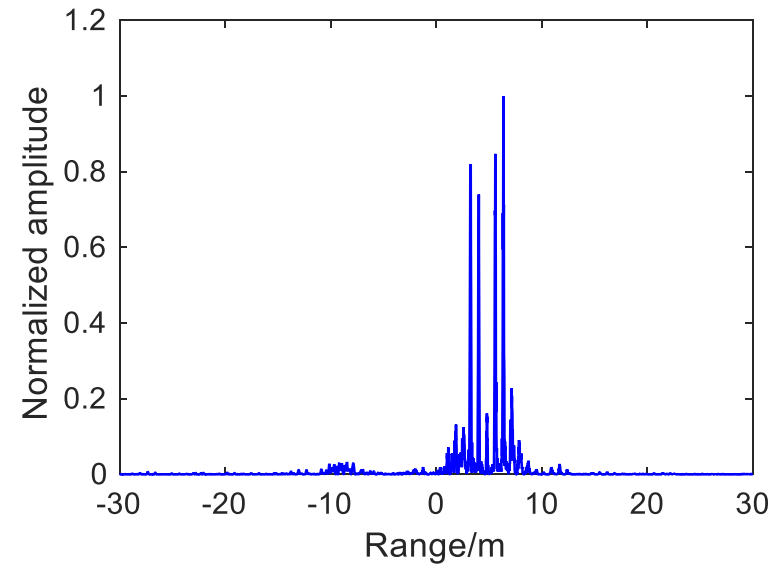

(b)

Fig. 13 Jamming suppression results, where $\tau_{d}^{n}=5.45 \mu$ s and $D_{r}=50 \%$. a HRRP of the received returns. $\mathbf{b}$ Suppression result 


\subsubsection{Performances versus duty ratio}

In the above three cases, the duty ratio $D_{r}$ all is $25 \%$. In the case of Fig. 8, when the duration $\tau$ is set as $10 \mu \mathrm{s}$ and $D_{r}=50 \%$, the jamming effect and suppression result are shown as Fig. 13a, b, respectively. It is demonstrated that the duty ratio mainly affects the amplitude of false target $[1,2]$. Our proposed method is also suitable for the case of large duty ratio.

\section{Conclusions}

Increasing attention has been paid to the ISRJ, which can greatly complicate the target detection and recognition of wideband radar. The novelty of this paper is that an efficient filter is designed and expanded to counter ISRJ based on the TF characteristic differences between target echo and ISRJ in the domain of TF spectrum, where the energy concentrates. Meanwhile, a feasible jamming suppression scheme is proposed. The jamming suppression effects on ISRJ are also analyzed with different orders, delayed time, SNR, JSR, and duty ratio. Numerous results demonstrate that the designed filter can effectively suppress the jamming and noise. Moreover, the received echo suppressed by our scheme is similar with the target's HRRP on the basis of high CC and large SJNR improvement factor. It is beneficial for the target detection and recognition of wideband radar.

\section{Acknowledgements}

The authors thank the associate editor Guolong Cui and the anonymous reviewers for their beneficial comments and suggestions that improve the quality of this paper.

\section{Funding}

This work was supported by the National Natural Science Foundation of China under grants 61471191 and 61501233, the Aeronautical Science Foundation of China under grant 20152052026, the Funding of Jiangsu Innovation Program for Graduate Education under grant KYZZ16_0169, and the Fundamental Research Funds for the Central Universities and this is partly funded by China Scholarship Council.

\section{Authors' contributions}

WX and GZ designed the jamming suppression scheme and experiments. $W X$ and WBL performed the experiments and analyzed the experiment results. WX and GZ contributed to the manuscript drafting and critical revision. All authors read and approved the final manuscript.

\section{Competing interests}

The authors declare that they have no competing interests.

\author{
Author details \\ ${ }^{1}$ Key Laboratory of Radar Imaging and Microwave Photonics, Ministry of \\ Education, Nanjing University of Aeronautics and Astronautics, Nanjing \\ 211106, People's Republic of China. 'Leihua Electronic Technology Research \\ Institute, Aviation Industry Corporation of China, Wuxi 214063, People's \\ Republic of China. ${ }^{3}$ College of Automation Engineering, Nanjing University of \\ Aeronautics and Astronautics, Nanjing 211106, People's Republic of China.
}

\section{References}

1. X Wang, J Liu, W Zhang, Q Fu, Z Liu, X Xie, Mathematic principles of interrupted-sampling repeater jamming (ISRJ). Science in China, Series F: Information Sciences 50(1), 113-123 (2007)

2. D Feng, H Tao, Y Yang, Z Liu, Jamming de-chirping radar using interrupted sampling repeater. Science in China, Series F: Information Sciences 54(10), 2138-2146 (2011)

3. S Gong, $X$ Wei, $X \mathrm{Li}, \mathrm{Y}$ Ling, Mathematic principle of active jamming against wideband LFM radar. J. Syst. Eng. Electron. 26(1), 50-60 (2015)

4. D Feng, L Xu, W Wang, $\mathrm{H}$ Yang, Radar target echo cancellation using interrupted-sample repeater. IEICE Electronic Express 11(8), 1-6 (2014)

5. W Wang, J Cai, A technique for jamming bi- and multistatic SAR systems. IEEE Geosci. Remote Sens. Lett. 40(1), 80-82 (2007)

6. W Wang, X Pan, Y Liu, D Feng, Q Fu, Sub-Nyquist sampling jamming against ISAR with compressive sensing. IEEE Sensors J. 14(9), 3131 3136 (2014)

7. X Pan, W Wang, G Wang, Sub-Nyquist sampling jamming against ISAR with CS-based HRRP reconstruction. IEEE Sensors J. 16(6), 1597-1602 (2016)

8. X Pan, W Wang, D Feng, Y Liu, Q Fu, G Wang, On deception jamming for countering bistatic ISAR based on sub-Nyquist sampling. IET Radar, Sonar and Navigation 8(3), 173-179 (2014)

9. X Pan, W Wang, D Feng, J Huang, Q Fu, G Wang, Rotational micro-motion modulated jamming for countering ISAR based on intermittent sampling repeater. Progress In Electromagnetics Research C 36, 41-56 (2013)

10. PE Pace, DJ Fouts, S Ekestorm, C Karow, Digital false target image synthesizer for countering ISAR. IEE Proc. Radar Sonar and Navigation 149(5), 248-257 (2002)

11. DJ Fouts, PE Pace, C Karow, SRT Ekestorm, A single chip false target radar image generator for countering wideband imaging radars. IEEE Solid-State Circuits 37(6), 751-759 (2002)

12. L Xu, D Feng, X Pan, Q Liu, X Wang, An improved digital false-target image synthesizer method for countering inverse synthetic aperture radar. IEEE Sensors J. 15(10), 5870-5877 (2015)

13. B Zhao, F Zhou, M Tao, Z Zhang, Z Bao, Improved method for synthetic aperture radar scattered wave deception jamming. IET Radar Sonar and Navigation 8(8), 971-976 (2014)

14. T Li, W Chen, G Lu, D Wang, A study on scatter-wave jamming for countering SAR, (Builin, Germany), in International Conference on Microwave and Millimeter Wave Technology, 2007, pp. 1-4

15. W Xiong, G Zhang, F Wen, J Yin, Trilinear decomposition-based spatialpolarizational filter method for deception jamming suppression of radar. IET Radar Sonar and Navigation 10(4), 765-773 (2016)

16. J Zhang, D Zhu, G Zhang, New antivelocity deception jamming technique using pulses with adaptive initial phases. IEEE Trans. Aerosp. Electron. Syst. 49(2), 1290-1300 (2013)

17. W Xiong, X Wang, G Zhang, Cognitive waveform design for anti-velocity deception jamming with adaptive initial phases (Philadelphia, USA), in 2016 IEEE Radar Conference, 2016, pp. 1-5

18. S Gong, X Wei, X Li, ECCM scheme against interrupted sampling repeater jammer based on time-frequency analysis. J. Syst. Eng. Electron. 25(6), 9961003 (2014)

19. MA Richards, Fundamentals of radar signal processing (McGraw-Hill Professional, New York, 2005), pp. 116-117

20. $X \mathrm{Li}, \mathrm{G} \mathrm{Bi}$, Systematic analysis of uncertainty principles of the local polynomial Fourier transform. EURASIP J. Adv. Signal. Process. 2014(1), 181 (2014)

21. G Yu, Y Zhou, General linear chirplet transform. Mech. Syst. Signal Process. 70-71, 958-973 (2016) 